\title{
NORMATIVA Y PROCEDIMIENTOS PARA LA PUBLICACIÓN DE ARTÍCULOS CIENTÍFICOS EN LA REVISTA DEL INTA.
}

\section{ASPECTOS GENERALES}

1. La edición de la revista es una de las actividades relevantes de Transferencia, por lo que se publicarán dos números por año (en junio y en diciembre).

2. La aceptación o no de los escritos será de acuerdo con las normas y procedimientos para publicar artículos científicos y es competencia del Comité Editorial.

Únicamente se aceptarán aquellos artículos que no hayan sido publicados en otra(s) revista(s).

3. La revista tiene carácter técnico - científico y en ella se publican:

a. Artículos científicos resultado de trabajos originales e inéditos de investigación (básica, aplicada), que a juicio del Comité Editorial tengan mérito científico o técnico.

b. Noticias técnicas (notas técnicas, avances de investigación, validación, etc.).

c. Análisis y comentarios sobre temas especializados.

d. Revisiones bibliográficas.

\section{PROCEDIMIENTOS.}

1. Una vez que el Comité Editorial recibe el artículo, dispone de un mes para enviarlo a los revisores. Los revisores internos tendrán un plazo máximo de un mes para entregar las publicaciones revisadas con un informe escrito de las mismas. A los revisores externos se les sugerirá el mismo tiempo para revisarlo.

Cuando el artículo es devuelto por los revisores, la editora o el editor dispondrán de ocho días hábiles para enviarlo a los autores con una nota en la que se indican las correcciones respectivas. Por su parte, los autores contarán con un plazo máximo de 15 días hábiles para hacer las correcciones y devolverlo, a la editora o al editor.

2. Los artículos científicos deben de tener una extensión máxima de 20 páginas escritas a doble espacio.

Las notas técnicas deben tener una extensión no mayor de 12 páginas escritas a doble espacio.

Se debe presentar un original y tres copias en hojas de papel bond tamaño carta y el texto grabado en un diskette preferiblemente de $31 / 2$ o CD de computadora, escrito en Word con letra Helvética No 12. Los gráficos y cuadros deben aparecer en Excel con sus respectivas tablas de valores.

3. En la redacción de los artículos se deben utilizar las Normas de la Real Academia de la Lengua Española, las unidades de medida del Sistema Métrico Decimal y las simbologías escritas de acuerdo con el Sistema Internacional de Unidades (SI) . Las unidades no llevan punto, se escriben con minúscula y no tienen plural (PCCMCA 2006).

Algunos ejemplos son: kilogramo (kg), gramo $(\mathrm{g})$, metro $(\mathrm{m})$, hectárea (ha), grados Celsius (C), milímetro $(\mathrm{mm})$, miligramo (mg) litro (I), metros sobre del nivel del mar (msnm) ,elementos ( $\mathrm{N}, \mathrm{P}$, entre otros), com-puestos químicos (como por ejemplo: $\mathrm{NaOH}, \mathrm{NaCl})$.

Cuando las unidades no están precedidas por un número, se expresan por su nombre completo sin utilizar su abreviatura. Por ejemplo: metro en lugar de $\mathrm{m}$. 
Los decimales se indican con coma; los miles con punto y los millones con un espacio.

En el caso de los números del cero al nueve, cuando no van seguidos de unidades, se escriben con palabra; y números para valores iguales o mayores a diez (UCR 1999).

4. Cuando los plaguicidas se citan en el título y texto se debe utilizar solo el nombre genérico del producto. Ejemplos: terbufos, cletodim. No se acepta el uso de nombres comerciales de plaguicidas .

\section{ESTRUCTURA DE LOS ARTÍ́CULOS.}

Título: tiene que ser claro, breve y conciso y lo más informativo posible. No más de 15 palabras es aceptable. Debe indicar el contenido del artículo de manera suficientemente explícita y precisa (MAG 1990).

Indicar con un "1", si el trabajo fue parte de una tesis, proyecto, etc.

En él se deben incluir los nombres científicos de las plantas y animales u otros organismos considerados en el estudio (UCR 1999).

Los nombres científicos (género, especie, cultivar y el nombre del clasificador) deberán ser citados para cada organismo en su primera mención, posteriormente se puede continuar usando el nombre vulgar. Se escriben con letra cursiva.

Autor(es): se considera ( $\mathrm{n}$ ) autor (es), el (los) individuo (s) (autor (es) personal (es), o la entidad (es), institución (es), asociación(es), organización (es), sociedad (es) autor(es) corporativo (s), responsable (s) de los contenidos intelectuales de las publicaciones.

El orden en el que se mencionan va de acuerdo con su contribución y aportes en la investigación y se colocan al margen superior derecho, debajo del título, nombre completo y un sólo apellido. Con una nota al pie de página indicando la institución para la cual labora el (los)autor (es). Se omiten los grados académicos del (los) autor (es).Si el (los) autor (es) lo desea (n) puede (n) indicar la dirección del correo electrónico.

Resumen: se coloca después del nombre de los autores y presenta en forma concisa el mensaje del artículo, describiendo brevemente los materiales y condiciones más relevantes del experimento. Debe indicar el año y lugar, los resultados obtenidos y las conclusiones más importantes. Las oraciones usadas deben ser racionales, objetivas y justificar el porqué de la investigación y el objetivo, evitando describir directamente los materiales y los métodos. La extensión no debe exceder de 250 palabras.

Palabras Clave : se deben incluir cinco palabras clave, las cuales deben ser diferentes a todas aquellas que aparecen en el título del trabajo (PCCMCA 2006).

Introducción: Define el problema que motiva la investigación y al final de esta sección se indican los objetivos o razones del estudio. Pueden incluirse citas bibliográficas para ayudar a la definición del problema y del trabajo. La extensión de ésta se recomienda sea de aproximadamente 350 palabras (MAG 1990). Toda información debe estar respaldad con citas bibliográficas.

Materiales y Métodos: describen en forma bien detallada la ubicación, la fecha de inicio y término, el ambiente, los materiales, las técnicas, los tratamientos, el diseño experimental, los análisis estadísticos y las variables a evaluar expuestos con suficiente claridad para que otros científicos puedan repetir el estudio. $\mathrm{Si}$ el método es muy conocido, solamente se incluyen referencias bibliográficas aclaratorias; si es nuevo o modificado se debe escribir nuevamente.En la redacción se debe seguir un orden cronológico (MAG 1990).

Resultados y Discusión: se recomienda que ambas partes vayan juntas, sin embargo, el autor podrá separarlas en caso que sea recomendable. Los resultados describen la información generada por la investigación; 
deben escribirse en forma concisa y siguiendo una secuencia lógica, usando cuadros y figuras (cuando se incluyen fotografías, mapas y dibujos entre otros, se les da el nombre de figuras y su numeración se debe ajustar a la secuencia de los gráficos). Los cuadros se presentan en tablas sin divisiones internas y externas. Los cuadros y figuras deben estar ubicados donde se mencionan, deben ser autoexplicativos y la información debe presentarse en forma completa, clara , de tal forma que no se tenga que recurrir al texto para entender el resultado presentado. Use decimales cuando sea justificado, si no, redondee 0 aproxime apropiadamente. Además de la descripción del contenido de la figura que se da en el nombre del título debe contener la provincia y el año en que se hizo el trabajo de investigación.

En la discusión no abuse de la estadística, úsela como una herramienta para probar la(s) hipótesis propuesta(s), con una base objetiva. Suministrar la significancia de las pruebas.

Se discutirán los resultados obtenidos, comparándolos con otros trabajos afines para dar interpretaciones o hacer deducciones lógicas sobre las diferencias o concordancias encontradas.

En la "Discusión" se debe explicar hasta qué punto los resultados obtenidos contribuyen a la solución del problema (limitantes) y qué puede traducirse en recomendaciones, aplicaciones, sugerencias, hipótesis, etc. (MAG1990).

Conclusiones: En las conclusiones se hace una síntesis de los resultados importantes producto de los datos obtenidos durante la ejecución del ensayo o experimento. Se resumen aquellos resultados sobresalientes obtenidos en la investigación realizada.

\section{REFERENCIAS BIBLIOGRÁFICAS (LITERATURA CITADA).}

La lista de la literatura citada debe estar conformada por no menos de diez citas bibliográficas y deben ser recientes.

Resumen de Normas Técnicas para la redacción de referencias bibliográficas

En IICA y CATIE (1999) se defíne la Referencia Bibliográfica como el conjunto de elementos suficientemente detallados que permiten la identificación de la fuente documental (impresa o no) de la que se extrae la información.

En términos generales esos elementos son: Autor, Año de publicación, Título y Subtítulo, Información sobre el documento, tal como notas tipográficas, volumen y número de revista, etc., y el orden y especificación varían según el tipo de documento: libro, revista serie, documento electrónico y otros.

A continuación se citan algunos ejemplos tomados de este documento. Si existiera dudas sobre la forma de citar los mismos, la información se encuentra en la dirección electrónica http:www.iica.int, luego dar clic en bibliotecas y aquí en Biblioteca Conmemorativa Orton y cuando aparece Servicios dar clic en Herramientas de Trabajo.

\section{Libros y Folletos.}

La portada es la fuente principal de la información para la redactar la referencia, sin embargo hay otras partes como la cubierta, la falsa portada, el colofón, la solapa, la introducción, etc.

Los elementos son:

Autor(es). Año de publicación. Título: Subtítulo. Mención del traductor y lo editor. Edición. Ciudad y/o país de publicación en caso necesario, Casa editora. Páginas o volúmenes (Mención de serie).

Crosby, PB.1990. Dinámica gerencial: el arte de hacer que las cosas ocurran. México, DF, Mc Graw-Hill.272p. (Serie de Administración).

\section{Tesis.}

Se elabora de la misma forma que la de los libros y folletos, pero después del título se anota la palabra Tesis seguida del grado académico en forma abreviada, en el idioma 
en que está escrita la tesis, Autor(es). Año de publicación. Título: subtítulo. Mención del grado académico. Ciudad y país donde se ubica la institución, Nombre de la institución que otorga el grado. Páginas.

Yah Correa, E. V. 1988. Crioconservación de suspensiones celulares embriogénicas de Musa spp iniciadas a partir de flores inmaduras. Tesis MSc. Turrialba, CR, CATIE. 77p.

\section{Conferencias, Congresos, Reuniones, etc.}

Los informes, memorias, actas, resúmenes de las conferencias, congresos, reuniones, simposios, nacionales e internacionales se anotan por el mismo nombre de la conferencia, congreso o reunión.

Los elementos son:

Nombre del evento (Número, Año de realización, Lugar donde se realizó). Año de publicación. Título. Mención del Editor (es). Ciudad y país de publicación, Casa editorial. Páginas o volúmenes.

Regional Workshop Needs and Priorities for Forestry and Agroforestry Policy Research in Latin América (1993 San José, CR). 1994.(Report). Eds.M Alfaro, R de Camino, M. I. Mora, P Oram. San José, CR, IICA. 298p.

\section{Analíticas.}

\section{A. Obra colectiva.}

Es la referencia biográfica de un trabajo escrito por un autor en un documento editado o compilado por otro(s) autor(es) tal y como es el caso de las conferencias, reuniones o congresos.

Los elementos son:

Autor, Año de publicación .Título del trabajo consultado. Preposición latina In, la referencia bibliográfica completa de la fuente que lo contiene, con las páginas iniciales y finales de la parte analizada sin mencionar nuevamente el año de publicación.
Mortimer, A.M.1990.Thebiologyofweeds. In Hance, JR; Holly,K. eds. Weed control handbook: principales. 8 ed. Oxford, GB, British Crop Protection Council. p. 1-42.

Santos Pereira, H dos. 1997. Brasil. In Reunión de los puntos focales de los Programas forestales nacionales de América Latina y el Caribe (1997, Brasilia, DF). Memoria. Santiago, CL. p. 49-56.

\section{B. Trabajo de un autor en su propia obra.}

La redacción de la referencia bibliográfica de una parte o capítulo con título específico escrito por un autor en una obra propia, tiene los elementos siguientes:

Autor. Año de publicación. Título de la parte o capítulo. Preposición In y los datos que incluye la referencia bibliográfica completa del libro o folleto sin mencionar nuevamente el autor ni el año de publicación. El autor se vuelve a mencionar en el caso que la publicación contenga más de un autor o un editor.

Phetig,R. 1994. Valuing the environmental methodological and measurement issues. In Ecological dynamics and the valuation of environmental change. Dordrecht, kluwer. p. 322.

Mugabe, J; Otieno-Odek,J. 1997. Nationalcaccess regimes: capacity building and policy reforms. In: Mugabe, J; Barber, CV; Henne,G; Glowka, L. eds. Access to genetic resources. Nairobi, ACTC. p. 95-41.

\section{Publicación periódica.}

Es aquella obra editada por lo general con título distintivo, en fascículos o partes a intervalos regulares, en orden numérico o cronológico y que pretende continuar indefinidamente. Incluye trabajos sobre temas diversos en un solo ejemplar, con la colaboración de varios autores (revistas, periódicos diarios). 


\section{A. Revistas.}

\section{Elementos:}

Autor(es). Año de publicación. Título del artículo. Nombre de la revista Volumen de la revista (Número de la revista): página inicial y final del artículo.

El volumen y el número se mencionan en números arábigos.

Singh,CK; Grewal, GS. 1998. Detection of rabies in central nervous system of experimentally infected buffalo calves. Indián Journal of Animal Sciences 68(12):12421254.

\section{a. Sin Volumen y sin número.}

Se recurre a algún elemento que pueda ayudar a su identificación, como son los meses o las estaciones del año.

Powles, H. 1987. Fencing off fish. Caribbean Farming feb.1987.13, 21.

\section{b. Con Volumen sin número.}

Si la revista tiene solamente el volumen se indica dicho dato, sin ninguna abreviatura.

Pierce, F. 1999. Aspects of precisión agricultura. Advances in Agronomy 67:1-58.

\section{c. Sin Volumen con número.}

Se utiliza la abreviatura "no." antes de dicho número.

Chamorro-Trejos, G. 1993. Zoca de café intercalada con nogal. Bosques y Desarrollo no.9:46-49.

\section{B. Periódicos o diarios.}

\section{Elementos:}

Autor(es). Año de publicación del periódico. Título del artículo. Nombre del periódico,
Ciudad de publicación, país abreviado, mes abreviado. Día: página.

Méndez, W.1998. Prometen apoyo a cooperativismo. La Nación, San José, CR, ene.8:6A.

\section{B. Separatas.}

La cita se hace según las normas establecidas para cada tipo de material. La fuente donde fue originalmente publicado el trabajo debe indicarse en una nota y en el idioma en que se redacta la bibliografía.

Sánchez, P. 1995. Science in agroforestry. Nairobi, ICRAF. 50 p. Reimpreso de:

Agroforestry Systems 30:5-55.

\section{Materiales cartográficos.}

Incluyen mapas o atlas de países, regiones, áreas y continentes; mapas o atlas básicos con datos estadísticos; estudios de observación en agricultura; cartas meteorológicas o hidrográficas, fotografías aéreas con fines cartográficos y otros.

\section{Elementos:}

Autor(es). Año de publicacción. Título. Edición. Lugar de publicación, Casa editorial. Escala. Paginación. Indicación de color (Serie).

Cortés, G. 1994. Atlas agropecuario de Costa Rica. San José, CR, EUNED. Ese. varía. 513p.Color

COSEFORMA (Cooperación en los Sectores Forestal y Maderero, CR). Convenio Costarricense Alemán. 1996. Zonas bioclimáticas de la región Huetar Norte de Costa Rica. San José, CR. Esc.1:200.000. Color.

\section{Material Audiovisual.}

Materiales gráficos (fotobandas, diapositivas, transparencias, fotografías, diagramas y otros) y colecciones de estos materiales; grabaciones sonoras (cintas, casetes, discos), microfichas, micropelículas, películas y videograbaciones. 


\section{Elementos:}

Autor(es). Año de publicación. Título: subtítulo. Mención del traductor y/o editor. Edición. Ciudad y país de publicación, Casa editora. Descripción física (Mención de serie).

\section{A. Microficha.}

FAO (Organización de las Naciones Unidas para la Agricultura y la Alimentación, IT).1990. Guidelines forsoil profile description (microficha). 2 ed. Roma. 10,5 x 14,5 cm.

\section{B. Diapositiva.}

CATIE (Centro Agronómico Tropical de Investigación y Enseñanza, CR). 1990. La investigación silvicultural (diapositivas). Turrialba, CR.110 diapositivas, son. ${ }^{+} 1$ casete ( 26 min.), color.

\section{Videocinta.}

Instituto para el Desarrollo de Sistemas de Producción del Trópico Húmedo de Tabasco,MX.1995. La mujer y la Agricultura.Tabasco, MX.(videocasete). 1 videocinta VHS(10:49 min.), son., color.

\section{Documentos Electrónicos.}

Actualmente en forma electrónica se encuentran monografías, publicaciones periódicas, mensajes, conferencias, reuniones, bases de datos, programas de computadora, etc. Por tanto, se seguirán las normas establecidas para cada uno de ellos y además se incluirán otros elementos que permitan identificar el medio en que están disponibles (en línea, disco compacto, disquetes, mensajes electrónicos, cintas magnéticas y otros.).

\section{Elementos:}

Autor(es). Año de publicación. Título: subtítulo.(Tipo de medio). Edición. Ciudad y país de publicación, Casa editora. Fecha en que se consultó el material para los documentos en línea. Descripción física. Disponibilidad y acceso para los documentos en línea. (Nota de serie).

\section{A. En línea.}

Documento disponible en línea a través de los servicios de internet.

\section{a. Libros.}

Guzmán, M de. 1993. Tendencias innovadoras en educación matemática, (en línea).

Bogotá, Unesco. Consultado 5 ene. 1998.

Disponible en

http://www.oel.org.co/oeivirt/edumat.htm.

\section{b. Revistas.}

Rodríguez, I. 1999.Tratamientos del agua potable, (en línea). Globo Terráqueo No. 20610. Consultado 10 set. 1999. Disponible en htttp://www.interbook.net/personal/ jigonzales1set99.htp

\section{c. Base de datos.}

Fundación Arias para la paz y el progreso humano, CR. 1998. Ceiba: base de datos ONG centroamericanas (en línea). San José,CR. Consultado 15 ene. 1998-Disponible en http://www.arias.or.cr./ceiba.

\section{d. Correo electrónico.}

Núñez,R.1999. Plan de trabajo SIDALC. (correo electrónico). Santo Domingo, RD, IICA.

\section{e. Disco compacto.}

Frater, H; Paulissen, D. 1995. El gran libro de multimedia. México, DF, Computec. 1 disco compacto, $8 \mathrm{~mm}$.

\section{f. En disquete.}

Los tamaños estándares comunes son: 5 1/4x8 pulgadas y $31 / 2$ pulgadas CIFOR (Centro para la Investigación Forestal Internacional,ID); Catie (Centro Agronómico Tropical de Investigación y Enseñan-za,CR).1997. Manejo del bosque natural latifoliado en el Trópico Americano. Turrialba, CR., Biblioteca Conmemorativa Orton. 1 disquete HD. 3"2 pulgadas (Serie Bibliotecología y Documentación. Bibliografía no. 26). 
Normativa y procedimientos para la publicación de artículos científicos en la revista INTA.

\section{Comunicaciones Personales.}

No deberían figurar en la literatura citada, se mencionan en nota al pie de página en el texto de la publicación.

\section{Elementos:}

Autor. Año en que tuvo lugar la comunicación Título de la comunicación. Lugar, e institución donde trabaja el autor. Mención de Comunicación personal.

Aguilar, JF. 1997. Forestería social (entrevista). San José, CR, Universidad de Costa Rica. Comunicación personal.

Salazar, F. 1999. Formación de consorcios (correo electrónico). Bogotá.Comunicación personal.

\section{Notas.}

Son datos suplementarios sobre el contenido o ciertas características especiales de un documento, que se agregan a la referencia para aclarar y ampliar información cuando es necesario.

Las hay de dos tipos : Notas de contenido y Notas sobre las características específicas de la publicación.

\section{A. Notas de contenido.}

Notas sobre las características específicas de la publicación.

\section{B. Trabajos sin publicar.}

Si un trabajo no se ha publicado o está en proceso de publicación, se agrega la frase En prensa o sin publicar.

Somarriba, E. 1997. Shade management in coffee and cocoa plantations. Agroforestry Systems. En prensa.

\section{Formas de citar las referencias bibliográficas dentro del texto.}

Por cuestiones de ética y derechos de autor todo investigador debe dar crédito de los trabajos que ha utilizado para desarrollar su investigación, facilitando con ello identificar a los autores de planteamientos y resultados anteriores que fundamentan dicha investigación.

Hay diferentes modos de citación en el texto que varían según las disciplinas. No obstante, en el caso de trabajos científicos y técnicos el que más se emplea es el Sistema autor-fecha. Consiste en referenciar un trabajo, del texto a la lista bibliográfica publicada al final de la publicación, por medio del apellido (s) del autor (es) seguido por el año de publicación.

\section{Cita contextual.}

En la redacción de cualquier trabajo de investigación se emplea con mucha frecuencia la cita contextual. La cita contextual es aquella en que un autor toma una idea, un resultado o un punto de vista de otro autor y lo presenta en sus propias palabras para reforzar o aclarar su propia investigación. Puede redactarse de dos maneras:

\section{Haciendo énfasis en el autor.}

Es cuando el nombre del autor va incluido en la redacción del párrafo.

Brenes (1998) ha demostrado que las variedades de mayor rendimiento son más susceptibles al ataque de nemátodos.

Estudios realizados por Brenes (1998) muestran que las variedades de mayor rendimiento son más susceptibles al ataque de nematodos.

\section{Haciendo énfasis en el texto.}

Es cuando se redacta el párrafo sin mencionar el autor. Este se indica entre paréntesis al final del párrafo. 
Las variedades de mayor rendimiento son más susceptibles al ataque de nematodos (Brenes 1998).

\section{Ejemplos con variaciones.}

\section{Publicación con un autor.}

Finegan (1992) demostró que el rendimiento... El mejoramiento genético da mejor rendimiento... (Finegan 1992).

Estudios realizados por Rivas Platero (1995) sobre micorrizas...

Avances de investigación en micorrizas... (Rivas Platero 1995).

\section{Publicación con dos autores.}

En el caso de dos autores de una misma publicación se cita por los apellidos de ambos unidos por la conjunción "y".

Rodríguez y Salas (1993) determinaron que la rentabilidad de los sistemas agroforestales... Considerando la rentabilidad de los sistemas agroforestales ... (Rodríguez y Salas 1993).

\section{Publicación con tres ó más autores.}

En el caso de tres o más autores de una misma publicación se cita por el apellido(s) del primer autor seguido por la expresión latina et al. (y otros).

Estudios realizados por Salazar et al. (1994) sobre la densidad de adultos virulíferos...

La densidad de adultos virulíferos de Bemisia...(Salazar et al. 1994).

\section{Más de una cita o publicación.}

Cuando se requiere citar más de una publicación a la vez, se debe separar cada una de ellas por coma (,). Las publicaciones deben mencionarse en orden cronológico por fecha de publicación, de la cita más vieja a la más reciente.
Ruíz (1980), García y Sánchez (1992) y Rojas (1996) analizaron muestras de suelos...

Fertilización con N, P, K aplicadas a muestras de suelos ... (Ruíz 1980, García y Sánchez 1992, Rojas 1996).

\section{Presentación, ordenación y organi-} zación de la lista bibliográfica.

Se recomienda presentarla al final del trabajo y se le asigna el título que más convenga:

"Literatura citada" o Literatura consultada".

Hay diversas formas de organizaría según el uso que se le vaya a dar; sin embargo en los trabajos científicos y técnicos predomina el arreglo alfabético por autor y en orden cronológico por año de publicación iniciando con la más antigua para finalizar con la más reciente.

\section{A. Citas de un mismo autor publicadas el mismo año.}

Luna, A. 1995a. El bosque protector. Mérida, VE, Instituto Forestal Latinoamericano.71p.

1995b. Ordenación sostenible de los bosques naturales en Venezuela. Criterio para la evaluación de la ordenación sostenible de los bosques tropicales: caso de Venezuela. Mérida, VE, Instituto Forestal Latinoamericano. $68 p$.

Si alguna de las citas de un mismo autor no tiene fecha de publicación, se coloca primero que las demás.

El nombre de un mismo autor (siempre y cuando sea el primero mencionado), no se repite en la lista, sino que a partir de la segunda referencia se sustituye su nombre por una línea de 8 espacios (__).

\section{B. Citas cuyo primer autor es el mismo, pero hay otros autores.}

Mesén, JF. 1993. Vegetative propagation of Central American hardwoods. Ph.D. Thesis. Scotland, University of Edinburgh. 230p. 
; Herasme, R.1996. Optimización de condiciones ambientales para la germinación de cedro (Cedrela odorata) y ciprés (Cupressus lusitanica Mill). Boletín Mejoramiento Genético y Semillas Forestales no.16:23-26. EEFBM (Estación Experimental Fabio Baudrit Moreno). 1999.

\section{LITERATURA CITADA}

UCR (Universidad de Costa Rica). 1999. Cómo presentar manuscritos. 1999. Revista de Biología Tropical 47(3):633639.

IICA (Instituto Interamericano de Cooperación para la Agricultura,CR); CATIE (Centro Agronómico Tropical de Investigación y Enseñanza,CR). 1999. Redacción de referencias bibliográficas: normas técnicas del IICA y CATIE.4ed. Costa Rica Biblioteca Conmemorativa Orton .25p.

MAG (Ministerio de Agricultura y Ganadería). 1990. Normas para la publicación de artículos científicos en la revista investigación agrícola. Investigación Agrícola 4(2):3-6.

Molestina, J. et.al. 1988. Fundamentos de comunicación científica y redacción técnica 1ed. Costa Rica. IICA. 267p.

PCCMCA (Programa Cooperativo Centroamericano de Mejoramiento de Cultivos y Animales). 2006. Agronomía Mesoamericana. 17 (3): 427-430. 
ALCANCES TECNOLÓGICOS, AÑO 4, NÚMERO 1 

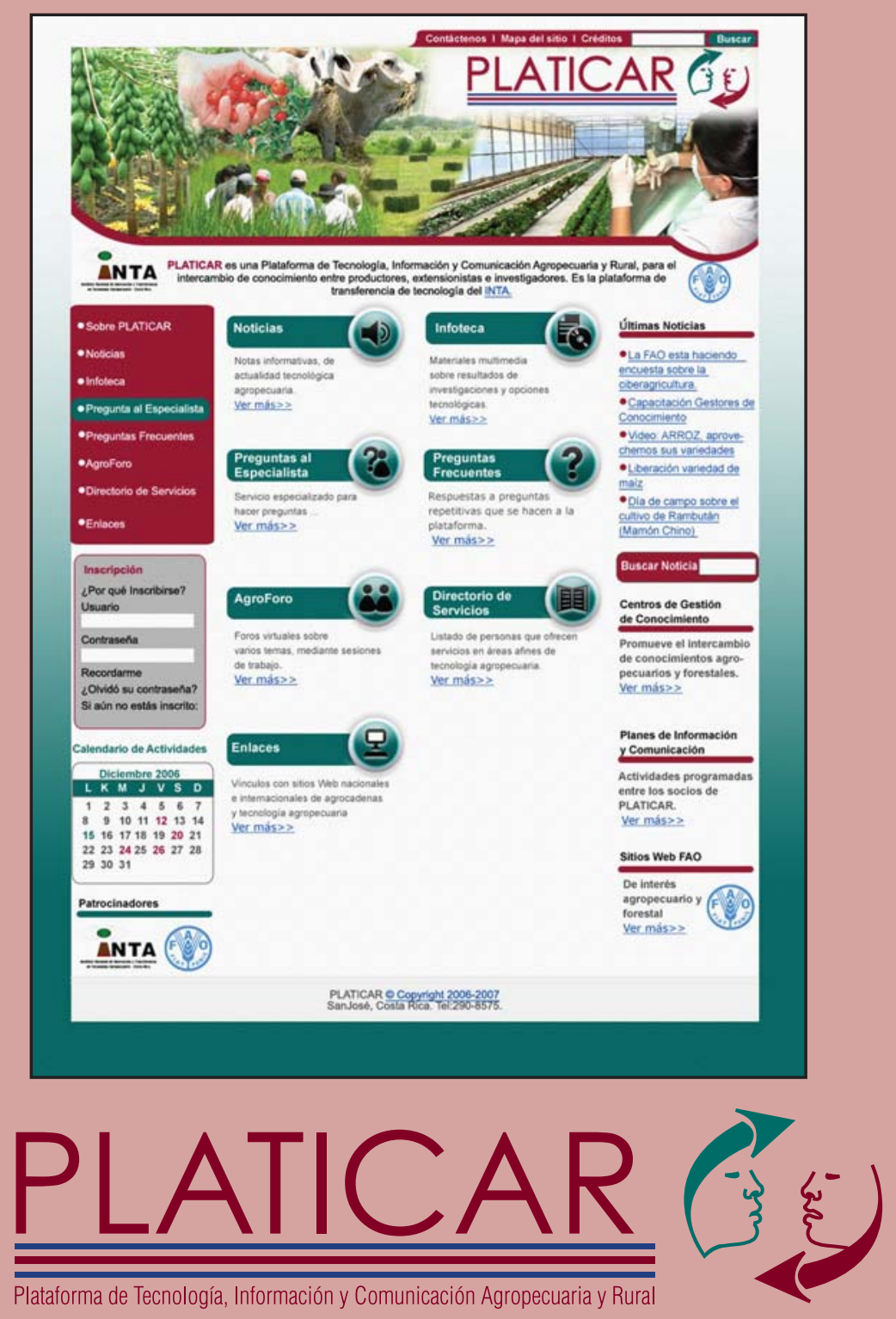

Es la plataforma de transferencia de tecnología del

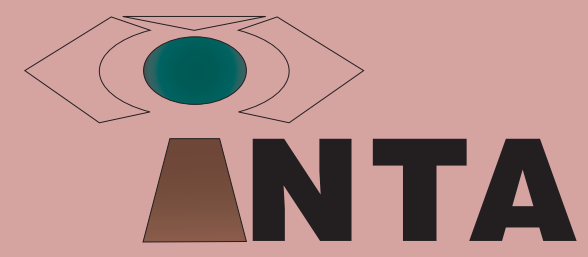

Instituto Nacional de Innovación y Transferencia en Tecnología Agropecuaria - Costa Rica

\section{www. inta.go.cr www.platicar.go.cr Tel.: 231-3991}

